\title{
Control of Hovering Altitude of a Quadrotor with Shifted Centre of Gravity for Inspection of High-rise Structures
}

\author{
Alexej Bulgakov ${ }^{a}$, Sergey Emelianov ${ }^{a}$, Thomas Bock ${ }^{b}$ and Daher Sayfeddine ${ }^{c}$ \\ ${ }^{a}$ South West University, Kursk, Russia \\ ${ }^{\mathrm{b}}$ Thomas Bock of TU Munich, Munich, Germany \\ ${ }^{c}$ South Russian State Polytechnic University, Novocherkassk, Russia \\ E-mail: a.bulgakow@gmx.de,ems@mail.ru,Thomas.Bock@bri.arch.tu-muenchen.de,daher@live.ru
}

\begin{abstract}
-
Unmanned aerial vehicles are specialized robots. Recently they were used in many civil applications such as patrolling, firefighting, rescuing tasks and being the shadow cinematographer in many movie-making companies. The new developments in material technology led to new sophisticated, miniature range of sensors and actuators, which are able to substitute gyroscopes and accelerometers. In light of this, new miniature aerial vehicles appeared in the market. Micro-UAV (MUAV) is able to infiltrate sites, where the bigger aerial robots could not. It is more maneuverable and recently is becoming able to fly to new altitudes. This allow the MUAV to be used for site scanning, to perform or assist in photogrammetry procedures in light to create 3D building models and maps. The future of the MUAV seems to be existing: In relation to medical field, the aerial robot is being used to perform radiation leakage test that, is done manually in most of the hospitals. It is also planned to be used to deliver lightweight goods within the city.

The versatility and adaptability of the miniature aerial robot creates new requirements for stability, maneuverability and speed. In this paper, we offer to study the quadrotor while inspecting high-rise structures using simulations. As the quadrotor is vertical take-off and landing vehicle, it is very important to analyze the effect of the altitude on the stability of in order to create control algorithm able to maintain the necessary hovering position of the quadrotor. A better stabilization leads to better scanning results and high quality captured images.
\end{abstract}

Keywords-
Inspecting structures, Quadrotor, Optimized Control System, Simulation.

\section{Background}

The success of implementation of unmanned aerial vehicles to perform military tasks has made an opening in civil markets for such specialized robots. While we cannot compare the evolution of UAVs with piloted planes, were aircrafts flew "alone" in the sky, the UAV designers are challenged to achieve better stability, offer sophisticated navigation options and honor several safety terms. These requirements have created a need to develop new control algorithm, recognition methods, path planning and generation solutions. In this regard, many researchers and hobbyists put lots of effort to create the best platform to simulate UAVs, study their aerodynamics, design new controllers and trajectory generators. The quadrotor consists of one of the favorite research platform, as it is reasonably affordable and technically easy to build and maintain.

\section{Purpose}

Quadrotors are take-off and landing rotorcraft. They are known for their high maneuverability. The quadrotor, like other aircrafts that belong to the rotorcraft class, flies by changing the rotational speed of one or several rotors rigidly fixed to the frame. These rotors are the only flying mechanism and lift force generator for such aerial vehicles. Although being described as a six degree of freedom object, threedimensional movement of the quadrotor is realized by four flight regimes only: hover, roll, pitch and yaw. The first being the only linear position to be controlled directly, the rest represents the rotational movement of the quadrotor. Recently, many approaches were 
adopted to simulate the quadrotor dynamics and control. These approaches consider the nonlinearity of the simulated system $[1,2,3,4,10]$. Most of the control schemes found refer to ideal position of center of gravity $[5,6,7]$. Using the ideal center of gravity in real control systems leads sometimes to tremendous errors in positioning. This is due to the following reason: the shifted center of mass generates additional accelerations and velocities that are registered by the inertial sensors meant to collect data on positioning. Figure1. illustrates possible real position of the center of gravity with reference to the quadrotor axis.

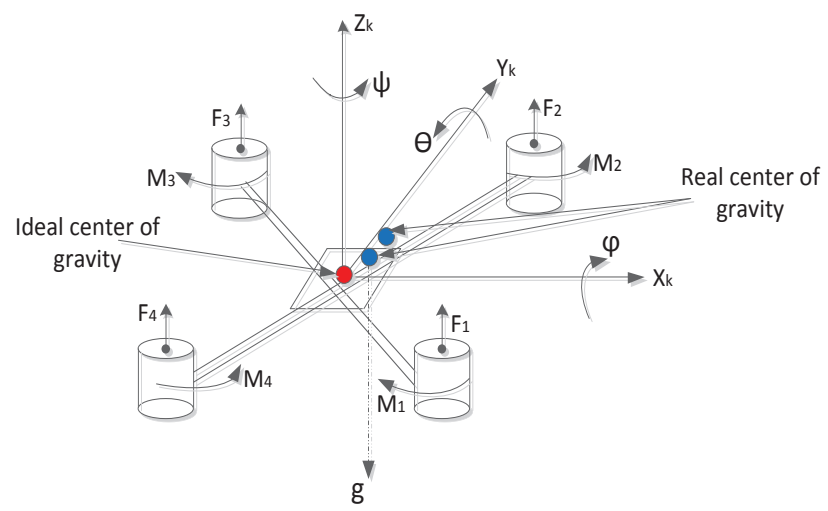

Figure1. Illustration of shifted position of centre of gravity.

Referring to the aforementioned analysis, we dedicate this paper to highlight the difference in considering an ideal and a real center of gravity in simulated control system. By achieving this, we will build our case study which consists of designing an optimized fuzzy logic controller to track a desired altitude, hereafter, to be implemented for scanning high-rise structures.

Inspection of buildings for cracks and damages is a long process, especially after natural disasters. It sometimes takes months to assess damages and compensate property owners. By automating this process using specialized robots, time is cut down tremendously. A swarm of quadrotors equipped with cameras can be used to achieve this quicker than doing it manually. Therefore, this specialized robot should be able to shoulder tasks optimally in positioning and time.

\section{Method}

To design an optimal control, it is mandatory to consider the nonlinearity of the quadrotor as well as the real position of the center of gravity. Quadrotor dynamics are described using the following equations $[5,7,8]$.

$$
\begin{gathered}
\ddot{x}=-\mathrm{c}(\psi) \mathrm{s}(\theta) \mathrm{c}(\phi)+\mathrm{s}(\psi) \mathrm{s}(\phi) \frac{T}{m} \\
\ddot{y}=-\mathrm{s}(\psi) \mathrm{s}(\theta) \mathrm{c}(\phi)-\mathrm{s}(\phi) \mathrm{c}(\psi) \frac{T}{m} \\
\ddot{z}=-\mathrm{c}(\theta) \mathrm{c}(\phi) \frac{T}{m}+g \\
\ddot{\phi}=\frac{T_{\phi}}{I_{x}} \\
\ddot{\theta}=\frac{T_{\theta}}{I_{y}} \\
\ddot{\psi}=\frac{T_{\psi}}{I_{z}}
\end{gathered}
$$

Where, $\mathrm{s}$ is Sine function, c is Cosine function, $\ddot{x}, \ddot{y}$ and $\ddot{z}$ are the second derivative (acceleration) of the quadrotor position along earth axis $\mathrm{OX}, \mathrm{OY}$ and $\mathrm{OZ}$ respectively and $\ddot{\phi}, \ddot{\theta}$ and $\ddot{\psi}$ are the second derivative of the roll, pitch and yaw angles, $I_{x}, I_{y}$ and $I_{z}$ are the terns of inertia of the quadrotor while performing a rotational movement, $\mathrm{m}$ is the mass of the quadrotor or term of inertia in linear movement and $T, T_{\phi}$ and $T_{\psi}$ are the total thrust generated per flight regime and its projections on the roll, pitch and yaw axis.

The quadrotor belongs to miniature rotorcraft class, which generally weighs more or less $1 \mathrm{~kg}$ and has a average length of $1 \mathrm{~m}$. We will shift the center of gravity position by $0.1 \%, 1 \%$ and $10 \%$ from the ideal position and check what influence it has on the performance of the autopilot $[4,9,10,11]$. The results (table.1) are obtained from simulation of 3D flight (XOYZ- earth axis) with $0,1 \%, 1 \%$ and $10 \%$ shifted center of gravity.

Table 1 Influence of shifted centre of gravity on ideally designed position controllers

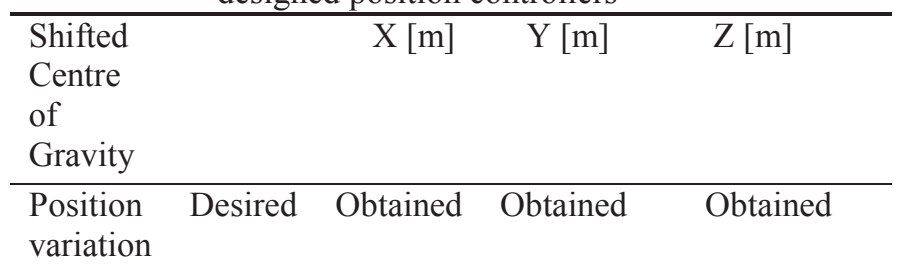




\begin{tabular}{lllll}
$0.1 \%$ & 1 & 1 & 1.053 & 1 \\
$1 \%$ & 1 & 1.009 & 1.12 & 1 \\
$10 \%$ & 1 & 1.996 & 2.218 & 1.003 \\
\hline
\end{tabular}

It is obvious from the results that by moving the center of gravity by $10 \%$, an error of $122 \%$ can occur by referring to one meter as desired position. This is due to the fact the control gains are calculated per different norms and parameters of the quadrotor. Considering this fact, with a different size and mass of quadrotor, the error in positioning can reach new records. Another aspect that can be noticed, it is the stability factor in altitude control, it is clearly seen from figure 2 that by increasing the variation coefficient by $1 \%$, the quadrotor tends to shake while hovering. In light of this, performing patrolling tasks such as scanning high-rise buildings, the quadrotor will be unstable due to windy conditions. Hence, it is necessary to design a control system able to keep the quadrotor in it position and track a desired trajectory.

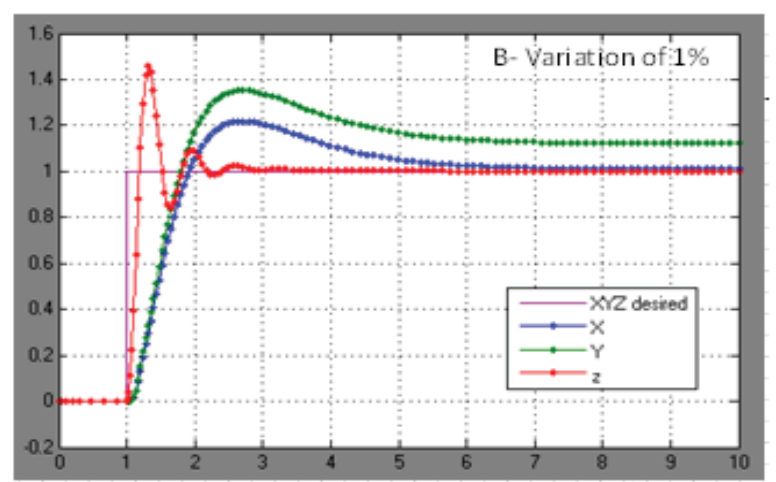

Figure 2. Simulation results of position control of a quadrotor with different shifting values of centre of gravity.

Horizontal axis- time [s], vertical axis position [m]. blue curve- OX position, green curve- OY position, red curve $-\mathrm{OZ}$ position.

To control the hovering altitude, we suggest a hybrid method consisting of fuzzy logic controller optimized using particle swarm algorithm. Although in many cases, artificial intelligence algorithms were ruled out from selection for such tracking and control tasks, due to the time consumption, the particle swarm method has pushed the ordinary fuzzy logic controller for new benchmark in stability and speed, as it will be proven in the listed simulation results. The fuzzy rules were obtained analytically by finding the resulting graph of the altitude error de(t) function with its derivative in time $f(e(t))$. The two inputs are represented using five triangular membership functions and the output of the regulator is represented using five triangular membership functions as shown in figure 3 a and $b$ (for inputs) and figure 4 (for output) respectively.

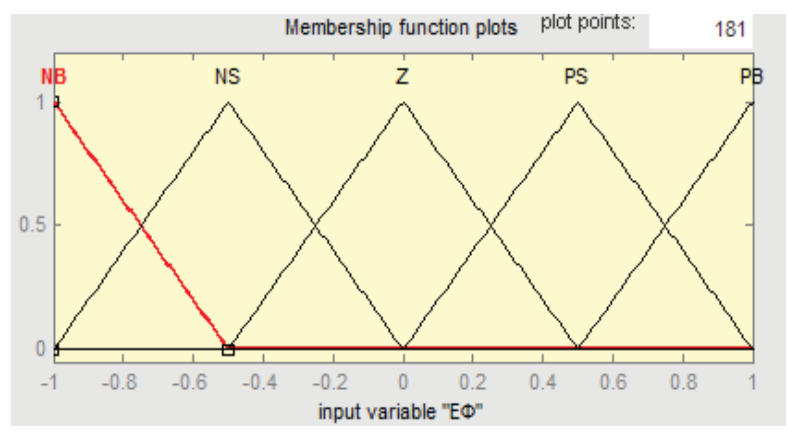

Figure 3. a- membership function for input "e(t)".

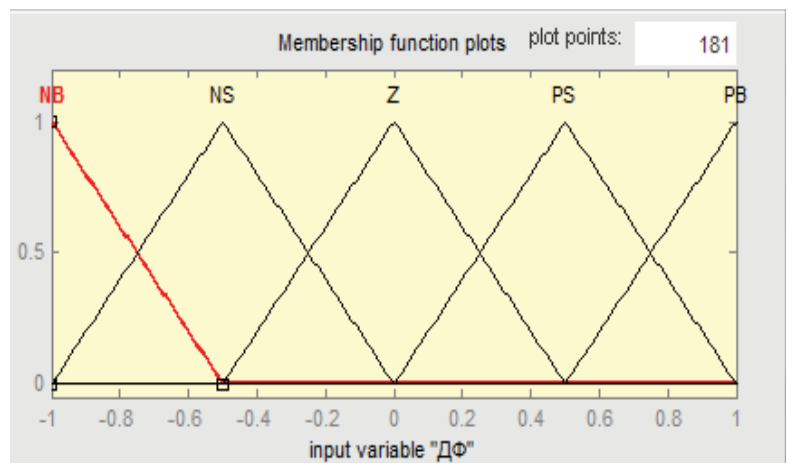

Figure 3. b- membership function for input "de(t)"

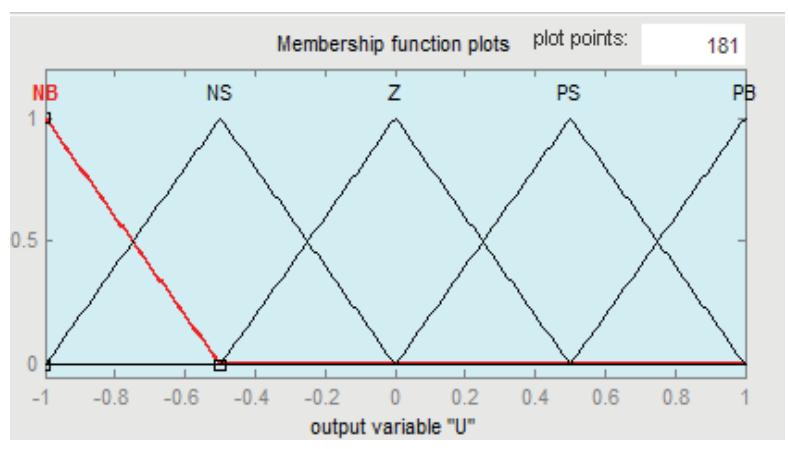

Figure 4. Membership function for fuzzy regulator output. 
The linguistic rules for the ordinary PD fuzzy regulator are listed in table (3).

Table 2 Calibration of membership functions

\begin{tabular}{|c|c|c|c|c|c|}
\hline$d e$ & NB & NS & Z & PS & PB \\
\hline NB & $(-1-, 1)$ & $(-1,-0.5)$ & $(-1,0)$ & $(-1,0.5)$ & $(-1,1)$ \\
\hline \multirow[t]{2}{*}{ NS } & $(-0.5,-$ & $(-0.5,-$ & $(-$ & $(-$ & $(-$ \\
\hline & 1) & $0.5)$ & $0.5,0)$ & $0.5,0.5)$ & $0.5,1)$ \\
\hline Z & $(0,-1)$ & $(0,-0.5)$ & $(0,0)$ & $(-0.5,0)$ & $(0,1)$ \\
\hline \multirow[t]{2}{*}{ PS } & $(0.5,-1)$ & $(0.5,-0.5)$ & $(0.5,0)$ & $(-$ & $(0.5,1)$ \\
\hline & & & & $0.5,0.5)$ & \\
\hline PB & $(1,-1)$ & $(1,-0.5)$ & $(1,0)$ & $(1,0.5)$ & $(1,1)$ \\
\hline
\end{tabular}

Table 3 Linguistic rules for fuzzy controller

\begin{tabular}{l|lllll}
\hline \multicolumn{1}{ll}{$d e$} & NB & NS & Z & PS & PB \\
\cline { 1 - 1 } & & & & & \\
NB & NB & NS & NB & NB & Z \\
Z & NS & NS & NS & Z & NS \\
PS & NB & NS & Z & PS & PB \\
PB & NS & Z & PS & PS & PS \\
\hline
\end{tabular}

Figure 5 represent generated plot of the output surface of a the fuzzy inference system using the first two inputs and the first output.

The particle swarm optimization will be used to tune the gain of the PD fuzzy controller.

The optimization method is a Runge -Kutta solver of differential equations. In particular, it uses six functions to estimate and calculate the fourth and fifth tolerance order. The difference between these solutions is taken as an error. This error estimate is very convenient to design adaptive algorithms, such as the use of fuzzy logic to control the process.

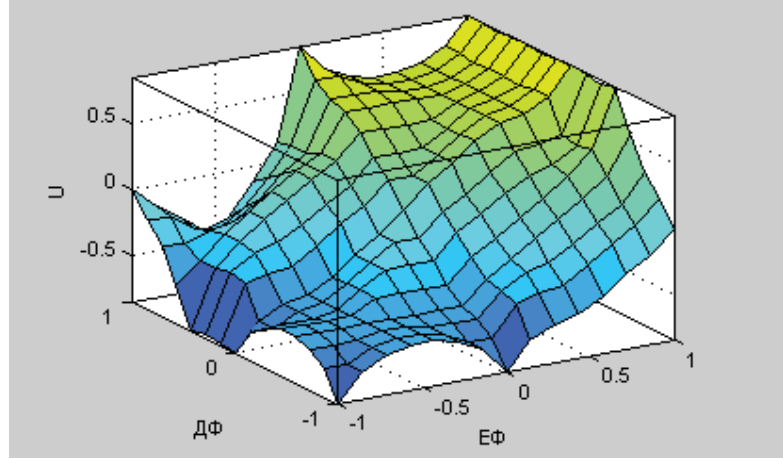

Figure 5. Fuzzy Interference Plot

The optimization algorithm works as follows: each particle in the swarm has a position. PSO determines the next best position the particle should migrate to, with reference to optimum criteria. The cycle continues until the particle reaches the most optimal position. Consequently, the system moves to

The global best value updating the swarm positions to the target. Equation (7) describes mathematically used PSO model

$$
\begin{array}{r}
V_{\mathrm{O \Pi}}^{k+1}=w V_{i}^{k}+c_{1}(k+1)\left(\text { pbest }_{i}-S_{i}^{k}\right) \\
+c_{2}(k+1)\left(\text { gbest }-S_{i}^{k}\right)
\end{array}
$$

Where, $V_{0 \Pi}^{k+1}$ is next value speed, w-weight function, $V_{i}^{k}$-current moving speed, $c_{1}, c_{2}$ are weights, pbest $_{i}$ is the personal best value for the particle, $S_{i}^{k}$ is the current position of the i-th particle and gbest is the global best position or the target [11].

\section{Results}

By implementing the obtained control scheme in altitude loop, the autopilot was able to maintain the quadrotor in hovering position. Figure. 3 shows simulation results of the altitude control using Fuzzy PSO method.

The comparison between the obtained results with those found in $[12,13]$ shows that the control approach in [13] (figure 7) lead to a satisfactory result by holding the quadrotor to hovering altitude of one meter with overshot equal to $0.6 \mathrm{~m}$ for 4 seconds. The time required for full stabilization is about 7 seconds. While the first curve (figure 6) shows that, the hybrid 
controller consisting of fuzzy logic regulator optimized using PSO has reduced the necessary time to reach onemeter altitude to 4 seconds and without overshot. Using the suggested algorithm, the quadrotor is more stable.

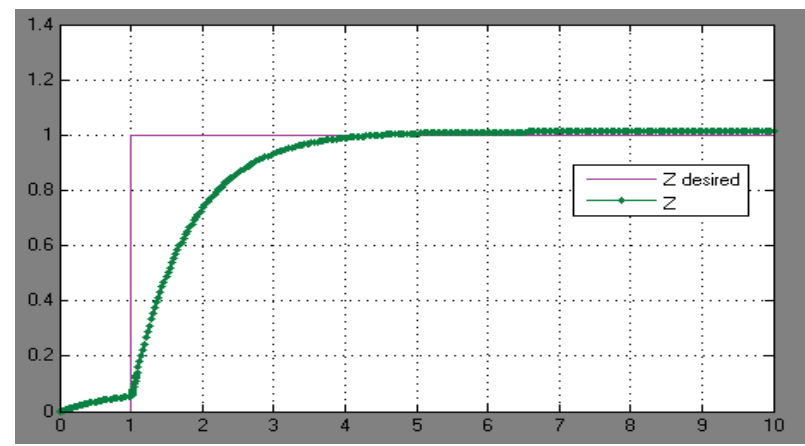

Figure 6. Simulation results of altitude control of a quadrotor with shifted centre of gravity using Fuzzy PSO. Horizontal axis- time [s], vertical axis- altitude [m].

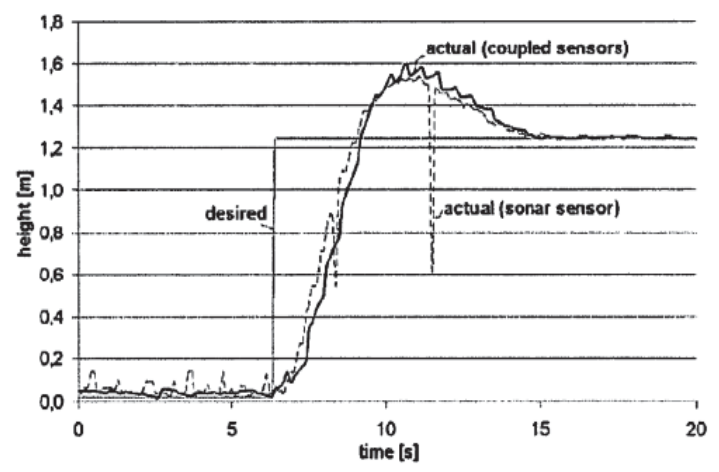

Figure 7. Altitude control results as by [13]. Horizontal axis- time [s], vertical axis- altitude [m].

\section{Implementation}

Building on the aforementioned results, we have changed the control task from hovering on an altitude of one meter, to scanning a sequence of building with different heights. Taking in consideration that the average height of one floor is about 3 meters; the desired altitude signal is designed to simulate 10storeyed building as minimum and 30 storied as maximum including the safety distance to be honored between the building and the UAV. This altitude is enough to challenge any miniature quadrotor (up to 1meter length) with drag effect, gravitational forces and peripheral wind force. The drag factor will consist a major competition to lift the quadrotor. The overall wind load on the quadrotor is calculated as follows

$$
F=S * P * C_{d} * K_{z} * G
$$

Where, $\mathrm{F}$ is the load of the wind on the quadrotor, $\mathrm{S}$ is the exposed area, $\mathrm{P}$ is the wind pressure, is the drag coefficient, is the exposure coefficient proportional to the elevation of the quadrotor from the ground and $\mathrm{G}$ is the gust response factor. Assuming that $\mathrm{S}, \mathrm{P}$ and are constants, and $\mathrm{G}$ will definitely changes in parallel with the altitude variation $\mathrm{H}$. The exposure coefficient ad the gust factor are calculated as follows. The exposure coefficient ad the gust factor are calculated as follows

$$
\begin{gathered}
K_{z}=(H / 33)^{2 / 7} \\
G=0.65+\frac{0.6}{(H / 33)^{1 / 7}}
\end{gathered}
$$

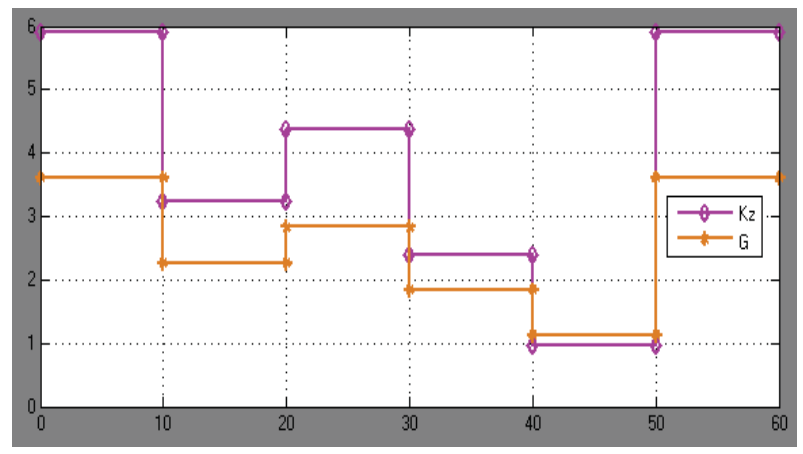

Figure 8. Simulation of $K_{z}$ and $\mathrm{G}$ with attitude Variation. Horizontal axis- altitude [m] , vertical axis$K_{z}$ and $\mathrm{G}$.

Fig. 8. shows how the exposure coefficient and gust factor changes with altitude. Applying the aforementioned condition in the quadrotor model, we obtain the results in figure.9. It clearly shows the suggested Fuzzy PSO controller was able to lead the quadrotor to scan high rise structures with ideal stability (no overshot registered theoretically) conserving the fact of shifted center of gravity of the $\mathrm{UAV}$ and changes in surrounding circumstances such as wind. 


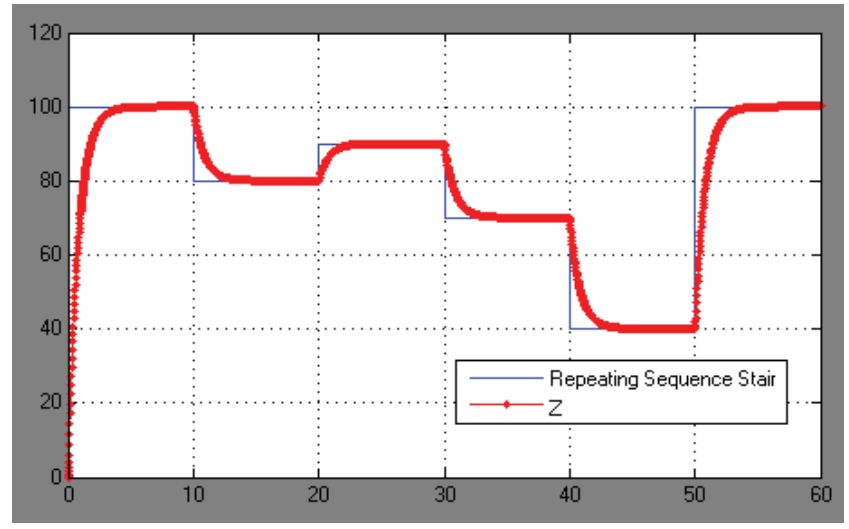

Figure 9. Altitude simulation results of high-rise inspection task. Horizontal axis- time [s], vertical axisaltitude [m].

\section{References}

[1] Isidori A. Nonlinear Control Systems, Springer- Verlag, , ISBN 3-540-19916-0. 1995

[2] Khalil, H.K.: Nonlinear Systems, PrenticeHall-New Jersey, 2002.

[3] Schwarz H.: Systems Theory of Nonlinear Control, Shaker-Verlag, ISBN 3-82657525-3. 2000

[4] Sayfeddine D. A comparative study between different techniques of controlling the roll angle and ox position of a miniature unmanned aerial vehicle // Технология 2013- часть 2.- С.107-109. 2013

[5] S. Bouabdallah. Design and control of quadrotor with application to autonomous flying. $\mathrm{PhD}$ dissertation. 2008

[6] Menno Wierema. Design, implementation and flight test of indoor navigation and control system of a quadrotor UAV.

[7] Tommaso Bresciani. Modeling identification and control of a quadrotor helicopter.
[8] Bouabdallah, S., Murrieri, P., Siegwart, R.: Design and Control of an Indoor Micro Quadrotor IEEE-International. Conference on Robotics and Automation, New Orleans, USA, pp. $4393-4398.2004$

[9] Bouabdallah, S., Noth, A., Siegwart, R.: PID vs LQ Techniques Applied to an Indoor Micro Quadrotor IEEE/RSj Internationad Conference in Intelligent Robots and Systems, September 28 - October 2 2004, Sendai, Japan. 2004

[10] Булгаков А.Г., Сайфеддин Д.., Караева Э.Р. Нелинейная интеллектуальная система управления квадрокоптером Шестая всероссийская мульти конференция по проблемам управления. МКПУ 2013. Том 2. Стр. 175-179. 2013 (In Russian)

Bulgakow A.G., Sayfeddine D., Karaeva E.R. Nonlinear Intellectual control system of a quadrotor. $6^{\text {th }}$ Multiconference on control problems MKPU 2013. Tom 2, pp. 175-179. 2013 (Translated)

[11] А.Г. Булгаков, Д. Сайфеддин. Интеллектуальное планирование траектории полета квадрокоптера // Известия Юго-Западного гос. ун-та. 2013. №5(50), C.54-62. 2013 (In Russian)

A.G. Bulgakow, D. Sayfeddine. Intellectual flight trajectory planning of a quadrotor. Proceedings of South-West University of Kursk 2013. №5(50), pp.54-62. 2013 (Translated)

[12] KEMPER M ; MERKEL M ; FATIKOW S. Attitude Control System for a Rotorcraft Micro Air Vehicle for Indoor Applications. UAVS-18th International Conference on Unmanned Air Vehicle Systems Conference,31 March 2003

[13] Kemper M. Control system for unmanned 4rotor- helicopter. Patent EP 1901153 A1. 2008 